\title{
MITIGATING THE MINDLESS MOUTHFUL THROUGH MODERATION, MANIPULATION, AND MINDFULNESS
}

\author{
LEXI ENDICOTT
}


The Boller Review, 2018

\section{ABSTRACT:}

The prevalence of global obesity is increasing at an unprecedented rate. Conventional weight loss programs, though logical in theory, usually prove to be ineffective in practice. There is an increased need and urgency for healthcare professionals to develop innovative methods to combat this pernicious health condition. Embracing the concept of mindful eating is one way that individuals can improve the quality of their health and well-being. This paper highlights the definition of mindful eating, describes the consequences of mindless eating, discusses the Japanese concept of hara hachi bu, and finally offers realistic practices that individuals can adopt in order to improve their connection with food and with others. 
Although Western civilization has realized remarkable achievements in science and medicine, the U.S. and other Western countries are experiencing unprecedented rates of diabetes, heart disease, and cancer. Epidemiological studies have shown that certain traditional societies practice patterns of behavior that significantly decrease the risks for such diseases. One of these societies is Okinawa, Japan, where individuals embrace the concept of hara hachi bua mindful approach to eating with Confucian roots wherein one eats to only $80 \%$ capacity. This mindset not only keeps one from eating excess calories, but it also emphasizes remaining mindful of one's connection to others, gratitude towards ancestors and the divine, and appreciation of the flavors, sights, and aromas of the meal. As an aspiring dietitian, my goal is to help individuals who struggle with food-related health issues recognize the beauty, simplicity, and power of mindful eating and the debauchery of the mindless mouthful. Adopting the practice of mindful eating into one's daily habits is a behavior that Americans should embrace in order to improve the quality of their mental, physical, and spiritual lives.

Before discussing mindful approaches to eating, I will first address why we should improve the way that we eat. According to the Centers for Disease Control and Prevention (CDC), heart disease is the leading cause of death in the United States, accounting for more than one in every three deaths. Cancer, stroke, and diabetes are also among the top ten leading causes of death (Price, et al.). All of these diseases lead to similar outcomes, such as decreased quality of life, increased healthcare costs, and a shortened lifespan. The more disquieting fact, however, is that they also all share a common cause: diet ("Why Good Nutrition is Important"). All of these diseases are strongly correlated with obesity, which, according to the World Health Organization, is defined as "abnormal or excessive fat accumulation that may impair health" ("Obesity and Overweight"). To truly understand how bad things are in the US, let us place our obesity rates in the context of global obesity rates. Although other countries experience obesity, the United States is, by far, in the worst condition: the latest data show that less than $4 \%$ of the Japanese population is obese. Canada reports an obesity rate of $25 \%$, the UK, $27 \%$, and Mexico, 33\%. Although these figures are certainly not optimal, the United States takes the cake (and apparently eats it too!). Over 38\% of adults in the United States are obese ("Health Status"). Even more concerning is that this rate is expected to rise to $47 \%$ by the year 2030 (Devaux, et al.).

The Academy of Nutrition and Dietetics acknowledges that the obesity epidemic is a "national public health priority" and is actively training registered dietitians to address this pressing issue (Schaefer and Zullo). The United States Department of Agriculture has released information on the status of Americans' suboptimal health and is actively pursuing solutions to decrease the negative consequences that accompany this issue. Currently, only $25 \%$ of Americans are consuming a diet containing adequate amounts of fruits, vegetables, whole grains, and healthy fats. Most Americans exceed recommendations for added sugar, saturated fat, sodium, and total caloric intake (U.S. Department of Health...). Thus, we can see that despite the well-meaning efforts of the USDA, publishing and promoting dietary guidelines is not inducing people to change their behavior.

I could go on and on about the deplorable state of Americans' expanding waistlines, but I will cut to the chase: a key fact about obesity is that it is preventable ("Obesity and Overweight"). If obesity is preventable, so are all of the adverse consequences that come with it. Traditional methods for weight loss include limiting total calorie intake, increasing fruit, vegetable, and whole grain intake, and increasing physical activity. This isn't news to anyone. We all know what to do to lose weight, but many people fail their diets, or more accurately, their diets fail them. So I am not here to prescribe the world's best diet to anyone. Instead, I am here to talk about an approach to eating that doesn't include calorie counting, restrictive meal plans, or strenuous exercise programs. I am here to discuss how integrating mindfulness into the rituals of daily eating can not only minimize one's midsection, but also augment the dining experience and improve one's connection to those with whom the experience is shared.

I would like to begin this discussion by first addressing the opposite of mindful eating: mindless eating. In his book, cleverly titled, Mindless Eating, Dr. Brian Wansink recounts dozens of studies that reveal shocking statistics that demonstrate how easy it is for us to ignore hunger and satiety cues. In one study, he invited college students to come into a restaurant lab for a free tomato soup lunch. Two of the four bowls of soup were designed so that they would discretely refill as the eater consumed the soup, essentially acting as bottomless bowls. After observing 62 participants, 
Wansink and his colleagues discovered that the participants eating from the bottomless bowls ate an average of $75 \%$ more soup than those given the normal soup bowls! Both groups reported the same levels of fullness, despite 100-200 calorie differences between the two (Wansink 50-52). This study revealed that people respond more strongly to environmental cues to stop eating than to signals from the mind or body, which certainly contribute to the ever-growing gut.

The inverse of mindless eating is, obviously, mindful eating. Although there are many definitions of mindful eating, it can simply be summarized as "the practice of enjoying food with understanding and compassion" (Fung et al.). This definition can broadly apply to all people regardless of age, gender, or health status. This approach to eating helps individuals increase their understanding, awareness, and compassion for their own body and mind, and their connection with others. Mindful eating begins with the practice of recognition, with the individual identifying when the body is hungry and when it is full. In the previously mentioned example, soup-eaters demonstrated an ignorance of these cues by consuming the tomato soup until they were told that their mealtime was over. Further, it includes cultivating a "nonreactive attitude to one's feelings toward food and eating" (Fung et al.). This element of neutrality allows the individual to appreciate the dining experience for what it is without harboring feelings of guilt or shame. The eater may acknowledge the inputs and outcomes of the event and then prepare for future interactions with food that will continue to increase their appreciation of the experience.

We can identify good examples of mindful eaters in the so-called Blue Zones. The Blue Zones are five areas in the world with the highest reported concentrations of centenarians, or people living over the age of one hundred. These places include Loma Linda, California (home of a large Seventh-Day Adventists society) Nicoya Peninsula, Costa Rica; Sardinia, Italy; Ikaria, Greece; and Okinawa, Japan. Not only are people living longer in these Blue Zones, but their quality of life, even after one hundred, is remarkable. Men still herd sheep through the countryside of Sardinia, Italy. Women leisurely and pleasurably work in their gardens. Men and women find ways to give back to their communities. Dan Buettner, author of The Blue Zones, discovered that all of these regions shared nine common principles that contributed to their long, fulfilled lives which he titled "The Power Nine" (264). Three of these principles address one's relationship with food: the $80 \%$ rule, "plant slant," and "wine at five." (Yes, you heard me correctly, wine consumption is correlated with a long, healthy life. Dr. Dennis said that advertising this fact would appeal to academics. But do remember, all things in moderation.) "Plant slant" emphasizes that the majority of one's diet comes from plant sources. Repeated studies have shown that there is a strong correlation between diets high in fruits, vegetables, legumes, and whole grains and lower body fat percentages and decreased inflammation (Jakse et al.). Plant-based diets also support animal welfare and environmental justice, and there is certainly a relationship between mindfulness, connectivity, and ecological equity, but for the purpose of my talk today, I will focus on the nutritional, rather than environmental, aspect.

What is fascinating about these cultures and their dietary habits is that what appears as mindful eating to us as Americans is simply eating to them. Expressing gratitude, eliminating distractions, and evaluating levels of hunger and fullness are simply habits that people in these cultures have practiced for centuries, thereby, effortlessly enhancing their eating experience. One of the most compelling rituals that individuals in Okinawa, Japan engage in is the practice of hara hachi bu.

Hara hachi bu is the Japanese concept of eating until one is only $80 \%$ full. Dan Buettner describes it as the "cultural practice of calorie restriction" (Buettner "Hara Hachi Bu"). Okinawans demonstrate this behavior by utilizing smaller serving vessels and by serving themselves, putting the food away, and then sitting down to enjoy the meal. This ritual deemphasizes the habit of "going back for seconds" (or thirds or fourths), and instead allows the individual to slow down, listen to her body, and engage with others at the meal. Americans can embrace the concept of hara hachi bu by implementing habits such as eating more slowly and intentionally, and by deliberately taking the time to listen to their bodies. We can eliminate distractions, such as TV, the internet, or our cellphones, all of which can hinder our ability to engage mindfully with the food and with others.

We can practice patterns of behavior reflective of the Okinawan lifestyle by increasing our awareness of our own body's cues. Dr. Wansink acknowledges that "there is a significant calorie gap between when an Okinawan says 
'I'm no longer hungry' and when an American says, 'I'm full'" (Wansink 34). "We gain weight insidiously, not stuffing ourselves, but eating a little bit too much each day - mindlessly" (Buettner 271). When we increase our awareness of our environment, express gratitude, and increase our perception of satisfaction, we decrease the need to be numbed by food. We can simultaneously enjoy consumption, conversation, and connection.

Although meditation and mindfulness often go hand in hand, there is an important distinction to make between the two. Meditation often involves sitting in contemplation, clearing the mind, and allowing thoughts to naturally come and go, like clouds in the sky. On the other hand, mindfulness revolves around objectively observing one's body, environment, and feelings. In mindful eating, it is not necessary for us to sit in the lotus position, clear our mind, or even watch our breath as we eat. Although these practices are useful, restorative, therapeutic, and could certainly be applied to mindful eating, they are not vital components for dining with increased awareness. Again, mindful eating is about bringing an increased sense of awareness to the body's natural cues and responding to these cues in an objective and nonjudgmental manner. For individuals just beginning on their journey of mindfulness, Nhat Hahn recommends "the method of pure recognition: recognition without judgment" (61). Begin the meal by recognizing how hungry or full you are. Recognize the inputs that contributed to the food being before you. Recognize the point at which you no longer feel hungry. As you develop the ability to recognize these signals, feelings of guilt and shame revolving around food will decrease. You will be able to be an example and a positive influence to those around you. When we are able to appreciate the eating experience through increased awareness, satisfaction, and simplification, those sharing the meal experience with us will be inspired to do the same. "Doing your best is the surest way to remind others to do their best" (Nhat Hahn 64).

There are many ways that you can adopt mindful eating principles and practices into your everyday lives. And although it may sound counterintuitive, adopting certain mindless habits can actually help you to eat more mindfully. Restructuring the eating environment is a key method in helping one to decrease the likelihood of careless consumption. Doing so can actually enable you to have more energy to focus on the more meaningful aspects of the meal such as connectivity and gratitude. Dr. Wansink states that "mindless eating habits can easily be changed, not by education, but by essentially changing the environment." Manipulation of our immediate environment can indeed change our behavior. Consider the following example: You notice that every day during your morning break, you grab four miniature chocolate bars from the candy dish, even if you don't feel hungry-it's simply a mindless habit. Without harboring any feelings of guilt, you acknowledge this behavior, decide that you wish to change this habit, and then reengineer your morning routine so that the chocolate is no longer a temptation. This may be accomplished by chewing gum during the break, saying aloud "I am going to eat this candy even though I'm not hungry," keeping a good distance between yourself and the candy dish during your break, or getting rid of the dish entirely. By mindfully and conscientiously reengineering the routine, the individual is then able to mindlessly eat better (or in this case, not eat, better). Adopting this new mindless habit could decrease calorie consumption by about 150 calories each day, which could prevent a potential 10-pound weight gain each year. Thus, we can see that mindfully restructuring the environment and our small habits can actually have a great impact on our overall wellbeing. "The best diet is the one that you don't know you're on"! (Wansink 1). We need not eliminate entire food groups or join intense weight loss programs in order to see positive changes. Rather, simple changes to your environment can bring gradual and sustainable benefits. But just as weight gain doesn't happen overnight, neither does weight loss. Time is required for effective weight loss, and patience and perseverance can actually help enhance the practice of mindfulness. Increased awareness of even the smallest positive changes in the body will contribute to an elevated appreciation for intentional mindfulness.

Just as we can restructure our habits so that we mindlessly eat more mindfully, we can, of course, mindfully transform them as well. There are four practices we can adopt to increase mindful connectivity while eating. The first component addresses what we eat. Making choices that are beneficial to the body, environment, and animals are key considerations in this first approach. Following a diet that is rich in plant-based, minimally processed foods decrease the risk for heart disease and create less environmental stress. The second component of mindful eating addresses the why of one's food choices. We are influenced to make decisions about what to eat based on many factors, such as 
personal values, beliefs, knowledge, culture, advertising, and availability. Understanding the reasoning behind food choices can prompt individuals to evaluate if their dietary habits are best for themselves and for others. Next comes the examination of how much one eats. This practice relates back to the Okinawan's tradition of hara hachi bu. When we eat in a state of mindfulness, we are able to be more observant of the quantity of food consumed, which can decrease overconsumption, thus decreasing the risk for becoming overweight. Finally, mindful eating allows us to examine how we eat. "Am I distracted? Am I in a rush? What flavors am I experiencing? With whom am I sharing the experience? Do I feel stressed, sad, angry, or happy?" When we are able to make these observations, we are able to connect back with ourselves, with those we may be with, and with those that helped bring the meal to the table (Fung, et al.).

Obesity. Observation. Okinawans. Oh, my! There are a myriad of ways that we all approach food every single day. These avenues are unique to each of us, and so there cannot be one prescriptive approach that we all can take to improve our relationship with food. However, by reflecting, observing, and assessing where we are individually, we can come to realize basic principles and approaches that may help to improve our complex relationship with the food that we consume and those with whom we share it. My hope is that you leave here today with the motivation and the resolution to take a moment of mindfulness before your next meal. Whether that be by offering gratitude, engaging with the environment, or heeding hunger and stopping at satisfaction, I am confident that you will discover an increased sense of well-being as you adopt these practices. Incorporating these rituals into our daily lives can motivate others to do the same. "If in one class, one student lives in mindfulness, the whole class is influenced" (Nhat Hahn 64).

If you don't remember anything else from my presentation today, I urge you to consider these seven words by best-selling author, Michael Pollan. "Eat food. Not too much. Mostly plants" (Pollan 1). This simple maxim concisely summarizes how we can avoid the mindless mouthful and live more focused, centered, and healthful lives. That's just some food for thought. Thank you. 


\section{References}

Buettner, Dan. The Blue Zones: Lessons for Living Longer from the People Who've Lived the Longest. National Geographic Society, 2009.

Buettner, Dan. "Hara Hachi Bu: Enjoy Food and Lose Weight with This Simple Japanese Phrase." Blue Zones, 26 Dec. 2017, bluezones.com/2017/12/hara-hachi-bu-enjoy-food-and-lose-weight-with-this-simple-phrase/.

Devaux, Marion, et al. Obesity Update 2017. Organization for Economic Co-Operation and Development, 2017, Obesity Update 2017, www.oecd.org/els/health-systems/Obesity-Update-2017.pdf?TSPD_101_R0=c712cf9f 9c46ac65ecdc814ed784f03bx5C000000 0000000000b30ded8affff00000000000000000000000000005a947a3d $0000 \mathrm{fcd} 180$.

Fung, T. T. et al. "An Expanded Model for Mindful Eating for Health Promotion and Sustainability: Issues and Challenges for Dietetics Practice." J Acad Nutr Diet, vol. 116, no. 7, 2016, pp. 1081-1086, doi:10.1016/j.jand.2016.03.013.

Health Status. OECD, 2017, Health Status, stats.oecd.org/index.aspx?DataSetCode=HEALTH_STAT\#.

Jakse, B. et al. "Effects of an Ad Libitum Consumed Low-Fat Plant-Based Diet Supplemented with Plant-Based Meal Replacements on Body Composition Indices." Biomed Res Int, vol. 2017, 2017, p. 9626390, doi:10.1155/2017/9626390.

Mathieu, J. "What Should You Know About Mindful and Intuitive Eating?" J Am Diet Assoc, vol. 109, no. 12, 2009, pp. 1982-1987, doi:10.1016/j.jada.2009.10.023.

Nhat Hans, Thich. The Miracle of Mindfulness: an Introduction to the Practice of Meditation. Translated by Mobi Ho, Beacon Press, 1976.

"Obesity and Overweight." World Health Organization, World Health Organization, Feb. 2018, www.who.int/mediacentre/factsheets/fs311/en/.

Pollan, Michael. In Defense of Food: An Eater's Manifesto. Penguin Books, 2009.

Price, Thomas E, et al. US Government Printing Office, 2017, p. 18, www.cdc.gov/nchs/data/hus/hus16.pdf\#019.

Schaefer, J. T. and M. D. Zullo. "Us Registered Dietitian Nutritionists' Knowledge and Attitudes of Intuitive Eating and Use of Various Weight Management Practices." J Acad Nutr Diet, vol. 117, no. 9, 2017, pp. 1419-1428, doi:10.1016/j.jand.2017.04.017.

U.S. Department of Health and Human Services and U.S. Department of Agriculture. 2015 - 2020 Dietary Guidelines for Americans. 8th Edition. December 2015. Available at https://health.gov/dietaryguidelines/2015/

Wansink, Brian. Mindless Eating: Why We Eat More than We Think. Hay House, 2011.

"Why Good Nutrition Is Important." Center for Science in the Public Interest, 21 Mar. 2016, cspinet.org/eating-healthy/why-good-nutrition-important. 